\title{
Recent Advances of Microfluidic Platforms for Controlled Drug Delivery in Nanomedicine
}

\section{Fikadu Ejeta (D)}

Department of Pharmaceutics and Social Pharmacy, School of Pharmacy, College of Medicine and Health Sciences, Mizan-Tepi University, Mizan-Aman, Ethiopia
Correspondence: Fikadu Ejeta

Tel +251910136034

Email fikaduejeta@gmail.com

\begin{abstract}
Nanomedicine drug delivery systems hold great potential for the therapy of many diseases, especially cancer. However, the controlled drug delivery systems of nanomedicine bring many challenges to clinical practice. These difficulties can be attributed to the high batch-to-batch variations and insufficient production rate of traditional preparation methods, as well as a lack of technology for fast screening of nanoparticulate drug delivery structures with high correlation to in vivo tests. These problems may be addressed through microfluidic technology. Microfluidics, for example, can not only produce nanoparticles in a wellcontrolled, reproducible, and high-throughput manner, but it can also continuously create three-dimensional environments to mimic physiological and/or pathological processes. This overview gives a top-level view of the microfluidic devices advanced to put together nanoparticulate drug delivery systems, including drug nanosuspensions, polymer nanoparticles, polyplexes, structured nanoparticles and therapeutic nanoparticles. Additionally, highlighting the current advances of microfluidic systems in fabricating the more and more practical fashions of the in vitro milieus for fast screening of nanoparticles was reviewed. Overall, microfluidic technology provides a promising technique to boost the scientific delivery of nanomedicine and nanoparticulate drug delivery systems. Nonetheless, digital microfluidics with droplets and liquid marbles is the answer to the problems of cumbersome external structures, in addition to the rather big pattern volume. As the latest work is best at the proof-of-idea of liquid-marble-primarily based on totally virtual microfluidics, computerized structures for developing liquid marble, and the controlled manipulation of liquid marble, including coalescence and splitting, are areas of interest for bringing this platform toward realistic use.
\end{abstract}

Keywords: microfluidic, nanomedicine, controlled drug delivery, nanocarriers

\section{Introduction}

Nanomedicine is a branch of medicine that aims to use nanotechnology - that is, the manipulation and manufacture of materials and devices with a diameter of 1 to 100 nanometers - to prevent disease and to image, diagnose, monitor, treat, repair, and regenerate biological systems. ${ }^{1}$ Medicine nanotechnology, termed nanomedicine, have provided essential new strategies for the evolution of pharmaceuticals and biotech products. ${ }^{2}$ Following ground-breaking discoveries in the use of nanoscale materials, ${ }^{3}$ nanomedicine has begun to play an important position in pharmaceutical studies and product development and has resulted in significant commercialization efforts. ${ }^{4}$ Nanoparticles (NPs) are the main fringe of the growing area of nanotechnology and are expected to conquer continual demanding situations of inefficient drug delivery strategies. ${ }^{5}$ In fact, nano-particulate drug delivery was discovered to 
effectively affect nanomedicine because of their small size, tunable chemical surface properties, high volume-tosurface ratio, and, basically, the potential to load active pharmaceutical ingredients and imaging agents. ${ }^{6}$ Furthermore, nanomedicine drug delivery mediums have been shown to improve the useful and favorable result or effect and reduce side-effects associated with drugs that have already received market approval, to enable new therapeutics, and to inspire further investigation of active biological products undesirable pharmaceutical properties that were previously thought to be incapable of development. $^{7}$

Microfluidic technologies employ nano and microscale fabrication techniques to develop highly controllable and reproducible fluidic microenvironments. ${ }^{8,9}$ Lead compounds with controlled physicochemical properties can be produced using microfluidics, characterized in a high throughput fashion, and evaluated in an in vitro biomimetic fashion for human organ organ-on-a-chip. ${ }^{8,10}$ The microfluidic generation has emerged as an effective device for the fabrication of microparticles with controlled morphologies and preferred properties because of its capacity to exactly control the emulsification procedure and generate monodispersed compound droplets in the microchannels. ${ }^{11}$ The ability to generate double emulsions containing one, two, three, or more quantities of droplets with extreme accuracy demonstrates the level of control offered by microfluidics. ${ }^{12}$ To exactly control the release of payloads, it is far important to put together polymeric debris with regarded sizes and size distributions, because the particle size strongly influences the payload release rate. ${ }^{13}$ By tailoring their inner structures, the loading of therapeutics into polymeric debris and the release of payloads also can be tuned. ${ }^{14}$ Co-delivery of multiple drugs can be attained through adjusting the size and number of internal compartments. ${ }^{14,15}$ Another method to control the release of payloads is with the aid of using the usage of stimulus-responsive substances to synthesize polymeric debris. ${ }^{16}$ After publicity of the environmental triggers, which include $\mathrm{pH}$, temperature or ionic strength, the debris goes through a physicochemical alternative after which it releases the payloads. ${ }^{17,18}$ Microfluidics offers advantages in terms of small size distribution with less polydispersibility index, higher encapsulation and loading efficiencies, better batch-to-batch uniformity, and easy scale-up possibilities. ${ }^{19}$ Interestingly, the preparation of microfluidic chips is simple and easy, leading to the economical production of nano-carriers. ${ }^{20}$ Various microfluidic chips have been fabricated to synthesize organic, inorganic, polymeric, lipid-based vesicular, and hybrid nanocarriers. ${ }^{21}$ Taking it all together, the microfluidic technology provides a potential platform for the quick synthesis of different novel drug delivery systems. ${ }^{22}$

The production methods of polymer microparticles have become increasingly important for applications such as controlled drug delivery, medical diagnostic tests, obtaining super-hydrophobic surfaces, optimum design of toughened polymeric composites and food technology. ${ }^{23}$ Polymeric microparticles are made using a variety of processes, including suspension or emulsion polymerization, solvent evaporation, spray drying, spraying a polymer solution through a small hole, and the Shirasu Porous Glass (SPG) membrane emulsification technique. Traditional production processes, on the other hand, have a number of disadvantages, including the fact that they take time, cause particle coalescence, and result in nonhomogeneous particle sizes and shape non-uniformity. ${ }^{24}$ The electrospray approach can be used to circumvent these constraints. Furthermore, the electrospray process has numerous advantages over previous methods, including minimal residue, the use of fewer solvents, cheap cost, and the use of high molecular weight polymers. ${ }^{25}$ The micro devices are comprised of two flow focusing junctions that work together to create double emulsions in a two-stage process. The aqueous phase is symmetrically pinched off at the first junction at low flow rates, forming monodisperse aqueous monomer plugs. The oil phase encapsulates liquids 1 and 2 at the second junction, generating double droplets of aqueous and monomer phases. The compound droplets then reach a third junction, where the channel cross-section is enlarged, causing them to take on spherical shapes. In the large section, mass conservation forces the droplets to slow down significantly, reducing the distances between successive droplets and thus reducing the distances between consecutive droplets, thus reducing the distances between consecutive droplets. ${ }^{26}$

Drug delivery technology advancements can improve pharmacological factors, including efficacy and bioavailability, resulting in the discovery and development of more effective drugs for better patient outcomes and quality of life. Fabrication quality controls, product batch-to-batch fluctuation, and the inability to obtain physiologically relevant test results in traditional in vitro prescreening platforms are all obstacles to nanoparticle drug delivery. ${ }^{10}$ Microfluidics has evolved from micro to nanoliter fluid handling to include a multidisciplinary approach 
that may be used for a wide range of applications. ${ }^{27,28}$ In comparison to traditional methods, microfluidics provides a mechanism to build highly controllable, reproducible, and scalable fabrication methods for nanoparticle synthesis. When compared to traditional in vitro culture methods, organ-on-chip microfluidic technology provides highly relevant organ-specific testing platforms capable of biologically relevant experimental time scales while employing a fraction of the sample and media volumes $^{29,30}$ The application of innovative microfluidic techniques to nanoparticle development processes may be able to address key challenges in nanoparticle drug carrier clinical translation. ${ }^{10}$

\section{Overview of Controlled Drug Delivery Systems}

The aim of controlled drug delivery is to deliver the drug to the exact anatomical location at the preferred rate and time to enhance the efficacy, pharmacokinetics, and bioavailability of the drug at the same time as preserving the minimum side effects. Due to a few specific characteristics (specific or even more than one dosing, perfect and targetspecific release, sustainable and controlled delivery, and mild facet effects) of the current microfluidic techniques, unparalleled possibilities exist to manipulate drug delivery. ${ }^{31}$ Controlled drug delivery systems help to improve the ease of administration of effective drugs and are pharmacokinetically superior for drugs formulated in this dosage form, including biological products and synthetic drugs. They enhance the amount released and that reaches the systemic circulation from the therapeutic drugs with the aid of increasing the uptake, preventing presystemic metabolism, retaining drugs at the safe therapeutic level, and lowering the side effects by targeting drugs to particular cells or tissues. ${ }^{12,32}$ Drug delivery may be controlled to release the active drug in the favored quantity that might allow individuals suffering from certain diseases to obtain reproducible, in urgent need, and tunable dosing at the desired time. ${ }^{33}$ Such a device permits correct regulation of dosage for favored effects, minimizes the associated side effects, minimizes the related side effects, and averts repeated drug administration or implantation of devices, ultimately increasing patient compliance. ${ }^{34-36}$ Such drug delivery strategies are elaborated in Figure 1.

Microfluidic technologies provide low-cost, simpleto-use platforms for fluid flow control. Emulsions generated in microfluidic systems have been employed in

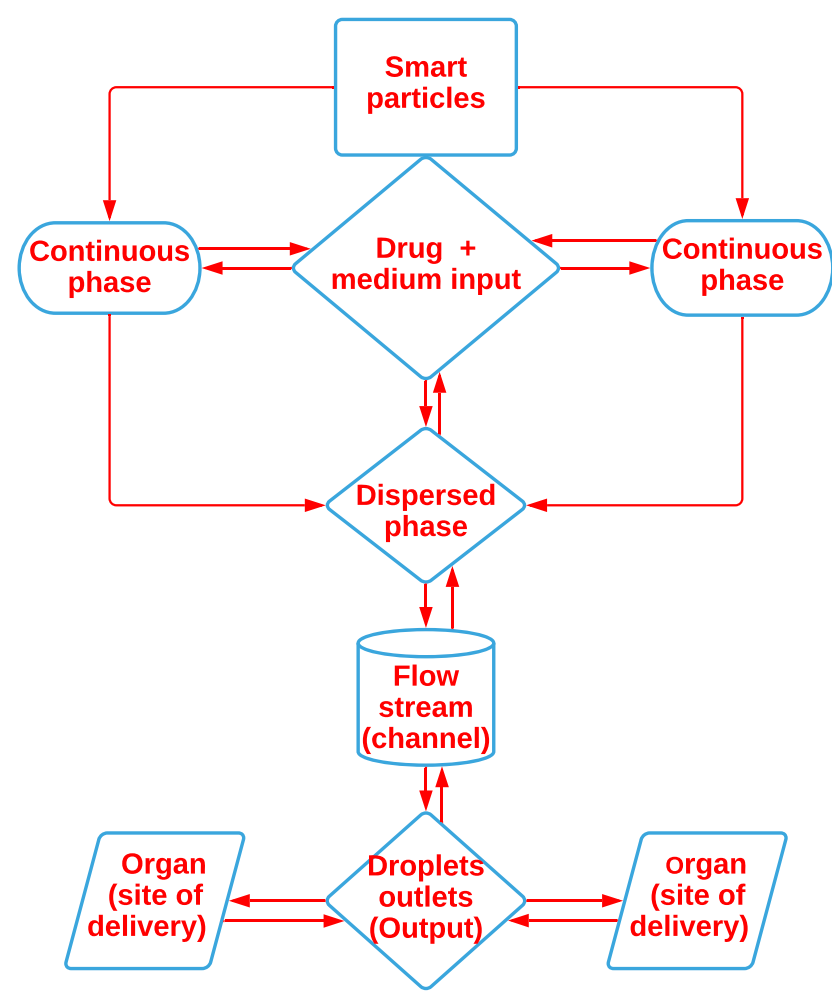

Figure I Microfluidics as a tool for drug delivery.

bioanalysis, organic synthesis, fluidic optics, and controlled drug delivery. ${ }^{12,32}$ T-junctions and flow-focusing nozzles are two types of devices used to generate emulsions in microfluidic platforms. ${ }^{37,38}$ Both approaches allow for the creation of monodisperse particles and provide versatility in the size of the emulsions produced. Monodisperse polymer particles, both spherical and non-spherical, are widely made by utilizing flow-focusing (FF) machines. It has been established that FF devices may be used to make photocurable polymeric particles, ${ }^{39,40}$ ioncrosslinkable thermosensitive gels, ${ }^{41,42}$ polymerencapsulated cells, ${ }^{4,44}$ and other particles. $^{21,45}$ The review by Brzeziński et al presented the utility of microfluidics technique for the preparation of polylactide (PLA)-based particles towards developing novel drug delivery systems. ${ }^{46}$ Brzeziński et al developed a novel microfluidic approach for the preparation of (co) polymeric and hybrid nanoparticles (NPs) composed of (co) polymers/tannic acid (TA) in the microfluidic flow-focusing glass-capillary device. ${ }^{47}$ However, there has been little systematic attempt to evaluate the controlled drug release kinetics from particles generated by microfluidic devices in most previous research. 


\section{Microfluidic Manufacture of Nanomedicines}

An extensive range of fabrication strategies has been explored and advanced for generating microfluidic elements and systems. ${ }^{48}$ Cast molding ${ }^{49}$ and associated soft lithography techniques, ${ }^{50}$ patterning of spin-on polymers $^{51}$ embossing $^{48}$ and combined methods are popular. $^{52-55}$

\section{Microfluidic Devices}

Microfluidic devices for particle production are mainly of two types, namely microchannels and microcapillaries. ${ }^{56}$ Microchannel-based devices are commonly fabricated by processes such as micromilling, micromachining, lithography, and mold replication. In such devices, the interfacial vicinity minimization brings about spontaneous droplet formation, and therefore, while preserving the oil-phase flow rate inside an optimum range, the droplet dimension is best depending on the microchannel geometry. Manufacturing of microchannel-primarily based totally overall devices is costly and takes a long time, but it allows for the fabrication of microsystems with particle size as small as a few tens of microns. Furthermore, in such devices, the microchannels may be flawlessly aligned, and in addition to uniform flow and highly liquefying of specific droplets or splitting droplets to uniform size, may serve to equip them, and the systems may be extended to produce a large number of products. Besides, capillary-primarily based structures are frequently made from low-cost components available in the market and have the capability to be microchannels in particle manufacturing. Importantly, the ones systems can be fabricated in a great deal much less time and can be operated in aggressive process conditions. ${ }^{57}$ In microchannel-based complete devices, the dispersed phase is in proximity to the tool's wall before being emulsified by the continuous phase, which may result in a phase inversion. ${ }^{58}$ While the affinity of the dispersed phase for matter is greater than that of the continuous phase, the dispersed phase preferentially wets the partitions of the tool. This turns the choice of the substance for the production of the device has superior importance to all others. Yet, phase inversion can be avoided via deciding on the proper device for aqueous or organic droplets. Preferentially, the polymers used may be changed on the vicinity wherein droplets of the dispersed phase are arise (develop); however, this interprets into a further step withinside the micro- fabrication process. $^{59}$ By contrast, capillary-primarilybased totally devices are tremendous for such terms. Here, the dispersed levels may be brought to the centerline of the non-stop segment flow, stopping the droplets from assembling the device's partitions. Furthermore, capillaryprimarily based totally devices do away with clogging troubles not unusual place in microchannel-primarily based totally devices and permit the manufacturing of oilin-water $(\mathrm{O} / \mathrm{W})$ or water-in-oil $(\mathrm{W} / \mathrm{O})$ emulsions with a unit microsystem. ${ }^{60}$

These developments have allowed microfluidic devices to be fabricated using a wide range of materials and geometries, enabling new and advantageous physical behaviors and qualities in microfluidic devices. For laminate microfluidic devices, each layer is cut individually. The cutting method has a significant impact on the dimensions and the device functionality. For prototyping and laboratory settings, cutting is usually done with a knife plotter (ie, xurography) or laser cutter because of the speed and simplicity that each tool offers. A knife plotter works by precisely cutting a material with a blade to create the geometry, while a laser cutter uses a focused beam (traditionally $\mathrm{CO} 2$ lasers are used). ${ }^{61,62}$ Under these conditions, the reduction of the diameter of droplets could be obtained by increasing flow rate, density ratio, and viscosity. ${ }^{63}$ Concerning the shape of devices used Bottom-up technologies that rely on emulsions or self-assembly cannot always provide fine, pre-designed control over particle geometry (shape, aspect ratio) and composition. ${ }^{64}$ Bicudo and co-workers investigated the manufacture of HANPs crosslinked with adipic dihydrazide $(\mathrm{ADH})$ and chloride carbodiimide utilizing a microchannel flow-focusing system $(\mathrm{EDCl})$. The work is focused on analyzing the process parameters of the unique method, which is a continuous nanoprecipitation at the water-organic solvent interface. The effect of the type of organic solvent employed, nonsolvent flow rate, and Hyaluronic Acid (HA) content on the characteristics of (hyaluronic acid nanoparticles) HANPs was investigated by the authors. ${ }^{65}$ The findings revealed that through water diffusion and nanoprecipitation rates, the affinity between water and organic solvent regulated the mean diameter of the nanoparticles (NPs). When the non-solvent exhibited an intermediate affinity for water, the polydispersity was narrowed. Furthermore, because the process was convection regulated, lower HA concentrations and higher isopropyl alcohol flow rates resulted in smaller particles. Regardless of the organic solvent, flow rate, or HA concentration, somewhat stable 
NPs were formed. The procedure was found to be straightforward, repeatable, and quick. The authors found that this process was promising for manufacturing oil-free HANPs, which are important for medical, pharmacological, and aesthetic applications. ${ }^{63,65}$ The benefits and drawbacks of these methods are mentioned in Table 1.

\section{Synthesis of Microfluidic Nanocarriers}

Beyond that, there has been great attraction of pharmaceutical formulators to synthetic nanocarriers and natural nanocarriers and colloidal systems have been of little interest. Significant attention has recently been paid to the production of organic nano-carriers, particularly in pharmaceuticals, as pharmaceutical scientists have begun to recognize the important properties conferred on nanocarriers by the microfluidic methods. ${ }^{21,72}$ In general, nanocarriers are obtained through the dispersion of preformed polymers or through the monomer reactions to form polymers. These nanocarriers may be advanced via unique strategies and are categorized into classes relying on processes involved. Emulsification of materials is required in the primary group, whereas it is not always required in the other groups. As a result, it provides a clean and simple method of synthesis. When those strategies are utilized in traditional device, lack of control over uniform blending, formation, and improved effects on formulation ingredients and, as a consequence, very few products have an excessive particle size distribution. On the other hand, microfluidics controlling structures are able to supply control over the above-mentioned elements as a consequence of giving them uniform size particles. ${ }^{73}$ Lipid polymer hybrid nanoparticles (LPHNPs) have been merged as capacity nanocarriers. ${ }^{66}$ LPHNPs were created with the help of the microfluidic co-flow nanoprecipitation method. ${ }^{22}$ The internal fluid changed into organized with the aid of using dissolving poly (lactic-co-glycolic acid) $(2 \mathrm{mg} / \mathrm{mL})$ into acetonitrile as a natural phase. ${ }^{74}$ The outer fluid contained lecithin and Distearoyl-sn-glycero-3-phosphoethanolamine-polyethylene glycol 2000 in a two-tothree mass ratio dissolved in $4 \%$ ethanol and answered in water. $^{21,75}$ These defined compatibilities, higher internalization, protection profile and a relative better anticancer interest within breast cancers with controlled drug release behavior. $^{21,22,73}$

Li et al conducted research on open-channel, water-inoil emulsification in a paper-based microfluidic drug delivery system. ${ }^{76}$ Providing novel open-channel microfluidics that is primarily based on surfaces with designed wettability and has the ability to control the floating of all excessive and occasional surface tension of liquids. The open channel devices have been capable of constraining numerous smaller surface tension oils at excessive, but sufficient flow rates to enable water-in-oil microfluidic emulsification in an open channel tool. It ought to extrude the dimensions of the emulsified droplets formed inside the open channel tool with the aid of adjusting the speed of each of the dispersed aqueous and natural continuous phases. Finally, a fabricated tool has been changed to be

Table I Advantages and Disadvantages of the Methods Used in in vitro Drug Screening by Microfluidics

\begin{tabular}{|c|c|c|c|}
\hline $\begin{array}{l}\text { In vitro } \\
\text { Culture }\end{array}$ & Advantages & Disadvantages & Reference \\
\hline 2D cell culture & $\begin{array}{l}\text { Cells grow on flat, usually plastic, dishes where they } \\
\text { adhere and spread, allow the study of cell behavior } \\
\text { using cheap materials and simple technologies, } \\
\text { universally known, several protocols and extensive } \\
\text { literature are available to analyze data and understand } \\
\text { cell behavior }\end{array}$ & $\begin{array}{l}\text { Restricted simulation of the complex cell-cell and cell- } \\
\text { matrix interactions to investigate cell behavior }\end{array}$ & [66-69] \\
\hline 3D cell culture & $\begin{array}{l}\text { Improves tissue organization; enhances the expression } \\
\text { of differentiated functions; and allows high-resolution } \\
\text { live imaging and high-throughput and low-cost drug } \\
\text { screening }\end{array}$ & $\begin{array}{l}\text { Static condition, difficult to completely mimic the } \\
\text { in vivo system and reflect their anatomic and } \\
\text { physiologic properties higher cell viability at the same } \\
\text { concentration }\end{array}$ & {$[10,70]$} \\
\hline $\begin{array}{l}\text { (Organ-on } \\
\text {-a-chip) }\end{array}$ & $\begin{array}{l}\text { System geometry and structures recreate physiological } \\
\text { length scales and concentration gradients, and the fluid } \\
\text { flow generates mechanical forces that recapitulate the } \\
\text { in vivo microenvironment }\end{array}$ & $\begin{array}{l}\text { Do not accurately predict human responses due to } \\
\text { inter-species difference in genetic makeup, along with } \\
\text { being extremely time-consuming, expensive, low- } \\
\text { throughput and raising ethical concerns }\end{array}$ & {$[29,7 \mid]$} \\
\hline
\end{tabular}


used efficaciously to synthesize surprisingly monodisperse hydrogel microparticles that might contain a drug molecule. Additional investigation of the drug delivery properties of the fabricated products had promising results with an open-channel microfluidic devices having the potential to achieve a high level of fluid manipulation while being manufactured quickly and cheaply. ${ }^{77}$ Another study conducted by Raphael and Martin utilized dynamic microfluidic method; dopamine was used as a model drug to quantify the electrochemical flow on paper-based devices. $^{78}$ They combined an electrochemical method with a microfluidic device for the time-resolved detection of dopamine from neuron-like PC12 cells cultured on filter paper. ${ }^{79}$ After an investigation of cellular adherence to the exterior of the paper with fluorescence microscopy; dopamine drug delivery after stimulation with acetylcholine without/with drugs has been investigated As a result, the data collected with the device agreed with single-cell statistics, demonstrating the technique's validity ${ }^{31}$ for higher-throughput quantification of chemical targets on tissues- or organs-on-a-chip. ${ }^{79}$ Generally, microfluidic devices maintain numerous qualities in the pharmaceutical sciences, which consist of suitable dosage, ideal drug delivery, site-targeted delivery, extended and controlled release, less repetitive dosing, and minimum side effects. These benefits are important qualities of drug delivery systems. Microfluidic techniques have been regularly employed in the production of polymers as carriers of many active moieties, direct drug delivery systems, highthroughput screening, and additives and excellent carriers of drugs. Cheaper and effortlessly produced paper-based materials are good substrates that truly mitigate several challenges associated with transportation, filtration, and storage, concentrators, valving, and multiplexing. ${ }^{31}$ Hereafter, paper-based microfluidic generation in controlled drug delivery programs might carry thrilling possibilities to amplify the frame of understanding within the subject and support the improvement of the scientific translation of drug delivery systems.

Huang et al prepared a stimuli-responsive controlled Vinblastine (VBL) drug release device from magnetically sensitive chitosan capsules. A magnetically responsive controllable drug delivery device has been designed by way of a means of embedding superparamagnetic iron oxide (SPIO) nanoparticles (NPs) in a chitosan matrix and an exterior magnet. In addition, droplet microfluidics, that is a completely unique technique for producing polymer spheres, has grown to be used for the manufacturing of monodispersed chitosan microparticles. The prepared VBL and SPIO NPs-loaded chitosan microparticles were characterized and showed individual and distinctive controlled-release patterns. Thus, the release rate, time, and dose of VBL release have become controlled through an exterior magnet. The outcomes presume that the usage of a magnetically responsive controlled drug delivery device brings a precious possibility for VBL drug release, wherein the delivery device is an energetic contributor, instead of a passive vehicle, within the optimization of most cancer treatments. This method actively focused magnetic drug delivery device to bring many benefits over traditional drug delivery structures through enhancing the precision and time of release, smooth operation, and better compliance for pharmaceutical applications. ${ }^{80}$ Because of their distinct physicochemical behavior and synergetic impact in preventing and inhibiting the progression of colorectal cancer, Liu et al chose atorvastatin and celecoxib as version dosage forms for their microfluidic collection of monodisperse multistage $\mathrm{pH}$-responsive polymer/porous silicon composites for exactly controlled multi-drug delivery. ${ }^{74}$ To be efficient in prevention and inhibition of the acceleration of colon and rectal cancer, the fabricated microcomposite incorporating hypromellose acetate succinate, which is not soluble in acidic conditions, however, is incredibly soluble in basic (alkaline) $\mathrm{pH}$ environments. ${ }^{81}$ The benefit of the larger pore volume of porous silicon (PSi) is atorvastatin was initially loaded into the PSi matrix before being encapsulated into $\mathrm{pH}-$ responsive polymer microparticles containing celecoxib through microfluidics to acquire multi-drug loaded polymer/PSi microcomposites. The manufactured microcomposites confirmed monodisperse size distribution, multistage $\mathrm{pH}$-response, particular ratiometric controllable loading extent closer to the concurrently loaded drug molecules, and tailor-made drug release kinetics of the loaded materials. This appealing microcomposite platform protects the payloads from being delivered at low $\mathrm{pH}$ values and improves the drug delivery at better $\mathrm{pH}$ values, which may be similarly used in preventing and treating colon and rectum cancer. Overall, the $\mathrm{pH}$-responsive polymer/PSi-primarily based completely microcomposites ${ }^{82}$ may be used as a common platform for the combined drug delivery system of various drug molecules. ${ }^{74}$

Monodisperse biodegradable polymer microparticles were prepared by $\mathrm{Xu}$ et al using a microfluidic flowfocusing tool for controlled drug delivery and have described the fabrication of monodisperse, drug-loaded 
microparticles from biodegradable polymers, the use of microfluidic flow-focusing devices and the drug-delivery properties of these particles.83 Particles are engineered in a variety of sizes ranging from $10 \mathrm{~mm}$ to $50 \mathrm{~mm}$. These particles are nearly monodispersed with a polydispersity index of $3.9 \%{ }^{84}$ Bupivacaine (amphiphilic) is included in the biodegradable matrix of the debris to characterize the formulation as a model drug. ${ }^{84-86}$ The kinetic evaluation suggests that the release of the drug from those monodisperse microparticles is slower than that from traditional strategies of identical average size, but reveals a larger particle size distribution and, more importantly, a substantial decrease in the primary burst than that discovered with traditional particles. ${ }^{84,86}$ The distinction in the preliminary kinetics of drug release is attributed to the uniform distribution of the drug within the particles generated with the assistance of microfluidic strategies. These outcomes showed the application of microfluidic flowfocusing on the technology of homogenous systems of particles for drug delivery. ${ }^{84}$

Meng and co-workers prepared thermal-sensitive liposomal controlled release by using a disposable microfluidic tool. The release of an encapsulated drug from a nanocarrier consisting of a liposome must increase local drug delivery while reducing the toxicity consequences of a temperature increase. ${ }^{87-90}$ High-intensity focused ultrasound (HIFU), particularly micro-HIFU (MHIFU) microfluidic devices, allows us to imitate the bulky HIFU transmission tool with the help of lower power consumption and control the release of low temperature-sensitive liposomes (LTSL) investigated. ${ }^{70,91}$ Furthermore, at the transition to a local temperature of between $41^{\circ} \mathrm{C}$ and $43^{\circ} \mathrm{C}$, the structure changes from a gel to a liquid crystalline phase, releasing the encapsulated drugs with an external source of hyperthermia, such as microwaves, and an infrared radiation laser with the structure of a lipid membrane of low-temperature sensitive liposomes (LTSL). ${ }^{32}$ The microfluidic era may also provide a promising approach to studying the complex dynamics of ultrasound and organisms at the ultramicroscopic level. ${ }^{92}$

A principal task within the improvement of polymeric nanoparticles for numerous programs is the specific engineering of preferred physicochemical properties in a reproducible manner. ${ }^{86}$ Shamsi and co-workers developed microfluidic self-assembly of polymeric nanoparticles with tunable compactness for controlled drug delivery. ${ }^{9,93}$ It is proven that the compactness of nanoparticles primarily based on self-assembled hydrophobically modified chitosan (HMC) biopolymers may be dictated by tunable fast blending through hydrodynamic flow focusing in microfluidic channels. It has been demonstrated through various flow rates, as well as the hydrophobicity of the chitosan chains, that the self-assembly properties of the chains can be controlled by optimizing the dimensions and compactness of the species, as well as a greater limited particle size distribution of the nanoparticles. ${ }^{86,94}$ The particle size of the formulation components increased with increasing blending time, while chitosan produced smaller and more compact nanoparticles with a much smaller variety of aggregated chains and a higher degree of hydrophobicity. The investigation revealed that, to the greatest extent possible, despite the lack of affinity for the aqueous medium and at blending times longer than the time of aggregation, nanoparticles with nearly equal forms of hydrophobic adhesion were formed. Furthermore, exploring the effectiveness of microfluidics directed to organizing HMCs and encapsulating paclitaxels, a common anticancer drug, has discovered remarkably higher encapsulation efficiency overall performance in comparison to the conventional bulk method. The invitro release of the paclitaxel from the synthetic nanoparticles was evaluated to analyze the effect of the compactness of the formulation components on the drug release properties. $^{37}$ The anticipated values of the diffusion coefficient of paclitaxel as high as 50\% at drug release implied sustainability in controlling drug release regarding the compactness of the nanoparticles, and an outstanding outcome as compared to the traditional bulk blending method. ${ }^{37,95}$ These outcomes suggest the excessive capability of the microfluidic method for specific bottom-up controlling of physicochemical properties of polymeric nanoparticles for numerous programs, ${ }^{96}$ which include controlled drug delivery. ${ }^{37,86,95}$

Electrokinetic microfluidic devices for rapid, low electricity drug release in self-sustaining microsystems evolved by Chung et al as a low electricity and strong electroactive microwell-primarily based totally implantable drug delivery system, meant to be used with selfsustaining microsystems, are presented. ${ }^{97}$ The tool has a top silicon-primary-based shape that describes the drug storage location and an electrically functionalized PDMS (polydimethylsiloxane) as a polymer. The drug release mechanism evolved right here exploits localized electrokinetic consequences of controlled drug release time and rate of chemical compounds saved in an unbiased, proper storage area. $^{97,98}$ It is proven how this could lessen the 
dosage time from hours to seconds over preceding diffusion, primarily based on the usage of as low as $20 \mathrm{~mJ}$ of strength in step with the dose. It turned into determined that the release technique can be completed in much less than $2 \mathrm{~min}$ or the usage of as low as $20 \mathrm{~mJ}$ of energy, each of which in comparison favorably to the state of the artwork microsystems. ${ }^{97}$ For the version of the electrokinetic delivery concerned with the inside of the release technique, detailed three-dimensional numerical simulations were used. The simulated model showed that much of the contents are released early from this technique. It further offers a physical point of view of the delivery process. ${ }^{20,97}$

\section{Future Perspectives and Challenges Microfluidic Drug Delivery}

Pattern production is required for many organic and chemical assays, hence mixing is an important stage in the production of microfluidic platforms. Diffusion-based amalgam techniques fall short of meeting the current demand for quick and uniform blending. Scholars have studied advances in crucial blending enhancement tactics, including blending with power sources, as well as difficult channel geometry. Real-time tracking and the capacity to mix with diverse continuous-flow properties are also advantages of continuous-flow microfluidic separation. An appropriate outside pressure for the group components can be chosen based on the particular signature of the group components, and an appropriate outside pressure may be selected for the separation process. Cutting-area advances in continuous-stream microfluidic separation strategies, which include magneto-fluidics, inertial microfluidics, acoustic-fluidics, dielectrophoretic, and optofluidics, have been developed. Emerging programs of mixed continuous-flow separation and combining technology for extra superior microfluidic platforms, including diagnostic and therapeutic micro bioreactors, lab-on-a-chip, and microfluidic chromatography for protein purification, have been investigated. Dropletprimarily based microfluidic strategies (DMF), including electrowetting-on dielectric (EWOD), dielectrophoresis, and magnetic strategies have been explained. Programs for more advanced combinatorial DMF devices have also been introduced. In addition, manipulation strategies for liquid marble as a microbioreactor have been demonstrated. Recent advances in microfluidics suggest that extra complicated microfluidic structures, specifically for blending programs, may be fabricated with three-D printing.

The design freedom provided by three-D printing will enable novel designs of nanomedicine formulations and preparations that were previously not possible with planar micromachining strategies such as soft lithography with poly-di-methyl-siloxane. Microfluidic cell way of life may be taken into consideration because of the next-technology method for biomedical and pharmaceutical programs. Liquid marble became as a promising digital microfluidics strategy. Continuous-flow microfluidics will remain used for programs that require excessive throughput. However, the trouble of cumbersome outside liquid transport and the requirement of optical microscopy for characterization makes continuous-flow microfluidics much less appropriate for programs with constrained pattern sizes. Digital microfluidics with droplets and liquid marbles is the answer to the problems of cumbersome outside structures, in addition to the rather big pattern volume. As the latest work is best at the proof-of-idea of liquid-marbleprimarily based on totally virtual microfluidics, computerized structures for developing liquid marble, and the controlled manipulation of liquid marble, including coalescence and splitting, are areas of interest for bringing this platform toward realistic use.

In summary, microfluidic technology allows for extremely precise liquid administration. It can be connected to an actuator system for on-demand or continuous drug release. Microfluidics has revolutionized the manufacture of drug carriers and the development of direct drug administration chips in general. Producing drug carriers that can generate a repeatable release profile as well as the controlled release of many compounds with varied release profiles needs the use of microfluidic technology.

\section{Disclosure}

The author reports no conflicts of interest in this work.

\section{References}

1. Viseu A. Nanomedicine. Encyclopedia Britannica, 23 Sep. 2020; 2021. Available from: https://www.britannica.com/science/nanomedicine. Accessed July 31, 2021.

2. Farokhzad OC, Langer R. Impact of Nanotechnology on drug delivery. ACS Nano. 2009;3(1):1-7. doi:10.1021/nn900002m

3. Juliano R. Nanomedicine: is the wave cresting? Nat Rev Drug Discov. 2013;12(3):171-172. doi:10.1038/nrd3958

4. Wagner V, Dullaart A, Bock AK, Zweck A. The emerging nanomedicine landscape. Nat Biotechnol. 2006;24(10):1211-1217. doi:10.1038/ nbt1006-1211 
5. Park K. Facing the truth about nanotechnology in drug delivery. $A C S$ Nano. 2013;7(9):7442-7447. doi:10.1021/nn404501g

6. Tomeh MA, Zhao X. Recent advances in microfluidics for the preparation of drug and gene delivery systems. Mol Pharm. 2020;17 (12):4421-4434. doi:10.1021/acs.molpharmaceut.0c00913

7. Riahi R, Tamayol A, Shaegh SAM, Ghaemmaghami AM, Dokmeci MR, Khademshosseini A. Microfluidics for advanced drug delivery systems. Curr Opin Chem Eng. 2015;7:101-112. doi:10.1016/j.coche.2014.12.001

8. Kwak B, Ozcelikkale A, Shin CS, Park K, Han B. Simulation of complex transport of nanoparticles around a tumor using tumor-microenvironment-on-chip. $J$ Control Release. 2014;194:157-167. doi:10.1016/j.jconrel.2014.08.027

9. Shamsi M, Zahedi P, Ghourchian H, Minaeian S. Microfluidic-aided fabrication of nanoparticles blend based on chitosan for a transdermal multidrug delivery application. Int $J$ Biol Macromol. 2017;99:433-442. doi:10.1016/j.ijbiomac.2017.03.013

10. Ahn J, Ko J, Lee S, Yu J, Kim YT, Jeon NL. Microfluidics in nanoparticle drug delivery; From synthesis to pre-clinical screening. Adv Drug Deliv Rev. 2018;128:29-53. doi:10.1016/j. addr.2018.04.001

11. Zhao X, Liu Y, Yu Y, et al. Hierarchically porous composite microparticles from microfluidics for controllable drug delivery. Nanoscale. 2018;10(26):12595-12604. doi:10.1039/c8nr03728k

12. Liu D, Zhang H, Fontana F, Hirvonen JT, Santos HA. Microfluidicassisted fabrication of carriers for controlled drug delivery. Lab Chip. 2017;17(11):1856-1883. doi:10.1039/C7LC00242D

13. Berkland C, King M, Cox A, Kim K, Pack DW. Precise control of PLG microsphere size provides enhanced control of drug release rate. $J$ Control Release. 2002;82(1):137-147. doi:10.1016/S0168-3659(02) 00136-0

14. Duncanson WJ, Lin T, Abate AR, Seiffert S, Shah RK, Weitz DA. Microfluidic synthesis of advanced microparticles for encapsulation and controlled release. Lab Chip. 2012;12(12):2135-2145. doi:10.1039/c2lc21164e

15. Araújo F, Shrestha N, Shahbazi MA, et al. Microfluidic assembly of a multifunctional tailorable composite system designed for site specific combined oral delivery of peptide drugs. ACS Nano. 2015;9 (8):8291-8302. doi:10.1021/acsnano.5b02762

16. Stuart MAC, Huck WTS, Genzer J, et al. Emerging applications of stimuli-responsive polymer materials. Nat Mater. 2010;9(2):101-113. doi: $10.1038 / \mathrm{nmat} 2614$

17. Zhao CX. Multiphase flow microfluidics for the production of single or multiple emulsions for drug delivery. Adv Drug Deliv Rev. 2013;65 (11-12):1420-1446. doi:10.1016/j.addr.2013.05.009

18. Mura S, Nicolas J, Couvreur P. Stimuli-responsive nanocarriers for drug delivery. Nat Mater. 2013;12(11):991-1003. doi:10.1038/nmat3776

19. Huang X, Lee RJ, Qi Y, et al. Microfluidic hydrodynamic focusing synthesis of polymer-lipid nanoparticles for siRNA delivery. Oncotarget. 2017;8(57):96826-96836. doi:10.18632/ oncotarget. 18281

20. Lin YS, Huang KS, Yang CH, et al. Microfluidic synthesis of microfibers for magnetic-responsive controlled drug release and cell culture. PLoS One. 2012;7(3):4-11. doi:10.1371/journal.pone.0033184

21. Karnik R, Gu F, Basto P, et al. Microfluidic platform for controlled synthesis of polymeric nanoparticles Rohit. Nano Lett. 2008;8 (9):2906-2912. doi:10.1021/n1801736q

22. Tahir N, Madni A, Li W, et al. Microfluidic fabrication and characterization of Sorafenib-loaded lipid-polymer hybrid nanoparticles for controlled drug delivery. Int J Pharm. 2020;581:119275. doi:10.1016/ j.ijpharm.2020.119275

23. Tasci ME, Dede B, Tabak E, et al. Production, optimization and characterization of polylactic acid microparticles using electrospray with porous structure. Appl Sci. 2021;11(11):1-13. doi:10.3390/ app11115090
24. Fantini D, Zanetti M, Costa L. Polystyrene microspheres and nanospheres produced by electrospray. Macromol Rapid Commun. 2006;27(23):2038-2042. doi:10.1002/marc.200600532

25. Xu Y, Hanna MA. Electrospray encapsulation of water-soluble protein with polylactide: effects of formulations on morphology, encapsulation efficiency and release profile of particles. Int $J$ Pharm. 2006;320(1):30-36. doi:10.1016/j.ijpharm.2006.03.046

26. Hennequin Y, Pannacci N, De Torres CP, et al. Synthesizing microcapsules with controlled geometrical and mechanical properties with microfluidic double emulsion technology. Langmuir. 2009;25 (14):7857-7861. doi:10.1021/la9004449

27. Whitesides GM. The origins and the future of microfluidics. Nature. 2006;442(7101):368-373. doi:10.1038/nature05058

28. Tokeshi M, Sato K. Micro/nano devices for chemical analysis. Micromachines. 2016;7(9):6-8. doi:10.3390/mi7090164

29. Huh D, Matthews BD, Mammoto A, Montoya-Zavala M, Hsin HY, Ingber DE. Reconstituting organ-level lung functions on a chip. Science. 2010;328(5986):1662LP- 1668. doi:10.1126/ science. 1188302

30. Bhise NS, Ribas J, Manoharan V, et al. Organ-on-a-chip platforms for studying drug delivery systems. J Control Release. 2014;190:82-93. doi:10.1016/j.jconrel.2014.05.004

31. Mao K, Min X, Zhang H, et al. Paper-based microfluidics for rapid diagnostics and drug delivery. $J$ Control Release. 2020;322:187-199. doi:10.1016/j.jconrel.2020.03.010

32. Meng L, Deng Z, Niu L, Cai F, Zheng H. Controlled thermal-sensitive liposomes release on a disposable microfluidic device. Proc Annu Int Conf IEEE Eng Med Biol Soc EMBS; November, 2015:5912-5915. doi: 10.1109/EMBC.2015.7319737.

33. Rhee MS, Galivan J, Wright JE, Rosowsky A. Biochemical studies on PT523, a potent nonpolyglutamatable antifolate, in cultured cells. Mol Pharmacol. 1994;45(4):783-791. PMID:7514264.

34. Sanjay ST, Dou M, Fu G, Xu F, Li X. Controlled drug delivery using microdevices. Curr Pharm Biotechnol. 2016;17(9):772-787. doi:10.2174/1389201017666160127110440

35. Sanjay ST, Zhou W, Dou M, et al. Recent advances of controlled drug delivery using microfluidic platforms. Adv Drug Deliv Rev. 2018;128:3-28. doi:10.1016/j.addr.2017.09.013

36. Chiang WL, Ke CJ, Liao ZX, et al. Pulsatile drug release from PLGA hollow microspheres by controlling the permeability of their walls with a magnetic field. Small. 2012;8(23):3584-3588. doi:10.1002/ smll.201201743

37. Thorsen T, Roberts RW, Arnold FH, Quake SR. Dynamic pattern formation in a vesicle-generating microfluidic device. Phys Rev Lett. 2001;86(18):4163-4166. doi:10.1103/PhysRevLett.86.4163

38. Nisisako T, Torii T, Higuchi T. Droplet formation in a microchannel network. Lab Chip. 2002;2(1):24-26. doi:10.1039/b108740c

39. Zhang H, Liu Y, Wang J, Shao C, Zhao Y. Tofu-inspired microcarriers from droplet microfluidics for drug delivery. Sci China Chem. 2019;62(1):87-94. doi:10.1007/s11426-018-9340-y

40. Nie Z, Xu S, Seo M, Lewis PC, Kumacheva E. Polymer particles with various shapes and morphologies produced in continuous microfluidic reactors. $J$ Am Chem Soc. 2005;127(22):8058-8063. doi:10.1021/ja042494w

41. Kim J-W, Fernández-Nieves A, Dan N, Utada AS, Marquez M, Weitz DA. Colloidal assembly route for responsive colloidosomes with tunable permeability. Nano Lett. 2007;7(9):2876-2880. doi:10.1021/n10715948

42. De Geest BG, Urbanski JP, Thorsen T, Demeester J, De Smedt SC. Synthesis of monodisperse biodegradable microgels in microfluidic devices. Langmuir. 2005;21(23):10275-10279. doi:10.1021/ la051527y

43. Tan WH, Takeuchi S. Monodisperse alginate hydrogel microbeads for cell encapsulation. Adv Mater. 2007;19(18):2696-2701. doi:10.1002/adma.200700433 
44. Clausell-Tormos J, Lieber D, Baret JC, et al. Droplet-based microfluidic platforms for the encapsulation and screening of mammalian cells and multicellular organisms. Chem Biol. 2008;15(5):427-437. doi:10.1016/j.chembiol.2008.04.004

45. Chang J-Y, Yang C-H, Huang K-S. Microfluidic assisted preparation of $\mathrm{CdSe} / \mathrm{ZnS}$ nanocrystals encapsulated into poly(DL-lactide-coglycolide) microcapsules. Nanotechnology. 2007;18(30):305305. doi:10.1088/0957-4484/18/30/305305

46. Brzeziński M, Socka M, Kost B. Microfluidics for producing polylactide nanoparticles and microparticles and their drug delivery application. Polym Int. 2019;68(6):997-1014. doi:10.1002/pi.5753

47. Brzeziński M, Socka M, Makowski T, Kost B, Cieślak M, Królewska-Golińska K. Microfluidic-assisted nanoprecipitation of biodegradable nanoparticles composed of PTMC/PCL (co)polymers, tannic acid and doxorubicin for cancer treatment. Colloids Surf $B$ Biointerfaces. 2021;201:111598. doi:10.1016/j.colsurfb.2021. 111598

48. Becker H, Gärtner C. Polymer microfabrication methods for microfluidic analytical applications. Electrophoresis. 2000;21(1):12-26. doi:10.1002/(sici)1522-2683(20000101)21:1<12

49. Jo BH, Van Lerberghe LM, Motsegood KM, Beebe DJ. Threedimensional micro-channel fabrication in polydimethylsiloxane (PDMS) elastomer. J Microelectromech Syst. 2000;9(1):76-81. doi:10.1109/84.825780

50. Xia Y, Whitesides GM. Soft lithography. Angew Chemie Int Ed. 1998;37(5):550-575. doi:10.1002/(sici)1521-3773(19980316) $37: 5<550$

51. Guerin LJ, Bossel M, Demierre M, Calmes S, Renaud P. Simple and low cost fabrication of embedded micro-channels by using a new thick-film photoplastic. Proceedings of International Solid State Sensors and Actuators Conference. Vol. 2; 1997:1419-1422. doi:10.1109/sensor.1997.635730.

52. Mescher MJ, Swan EEL, Fiering J, et al. Fabrication methods and performance of low-permeability microfluidic components for a miniaturized wearable drug delivery system. $J$ Microelectromech Syst. 2009;18(3):501-510. doi:10.1109/JMEMS.2009.2015484

53. Fiering J, Mescher MJ, Leary Swan EE, et al. Local drug delivery with a self-contained, programmable, microfluidic system. Biomed Microdevices. 2009;11(3):571-578. doi:10.1007/s10544-008-9265-5

54. Schomburg WK, Ahrens R, Bacher W, Martin J, Saile V. AMANDA - surface micromachining, molding, and diaphragm transfer. Sens Actuators a Phys. 1999;76(1-3):343-348. doi:10.1016/S09244247(98)00292-1

55. Rogge T, Rummler Z, Schomburg WK. Polymer micro valve with a hydraulic piezo-drive fabricated by the AMANDA process. Sens Actuators a Phys. 2004;110(1-3):206-212. doi:10.1016/j. sna.2003.10.056

56. Zhao X, Bian F, Sun L, Cai L, Li L, Zhao Y. Microfluidic generation of nanomaterials for biomedical applications. Small. 2020;16 (9):1-19. doi:10.1016/j.sna.2003.10.056

57. Herranz-Blanco B, Ginestar E, Zhang H, Hirvonen J, Santos HA. Microfluidics platform for glass capillaries and its application in droplet and nanoparticle fabrication. Int J Pharm. 2017;516(12):100-105. doi:10.1016/j.ijpharm.2016.11.024

58. Pessi J, Santos HA, Miroshnyk I, Joukoyliruusi J, Weitz DA, Mirza S. Microfluidics-assisted engineering of polymeric microcapsules with high encapsulation efficiency for protein drug delivery. Int $J$ Pharm. 2014;472(1-2):82-87. doi:10.1016/j.jpharm.2014. 06.012

59. Olanrewaju A, Beaugrand M, Yafia M, Juncker D. Capillary microfluidics in microchannels: from microfluidic networks to capillaric circuits. Lab Chip. 2018;18(16):2323-2347. doi:10.1039/c81c00458g

60. Martins JP, Torrieri G, Santos HA. The importance of microfluidics for the preparation of nanoparticles as advanced drug delivery systems. Expert Opin Drug Deliv. 2018;15(5):469-479. doi:10.1080/17425247.2018.1446936
61. Gale BK, Jafek AR, Lambert CJ, et al. A review of current methods in microfluidic device fabrication and future commercialization prospects. Inventions. 2018;3(3):60. doi:10.3390/inventions3030060

62. Niculescu AG, Chircov C, Bîrcă AC, Grumezescu AM. Fabrication and applications of microfluidic devices: a review. Int J Mol Sci. 2021;22(4):1-26. doi:10.3390/ijms22042011

63. Chiesa E, Dorati R, Pisani S, et al. The microfluidic technique and the manufacturing of polysaccharide nanoparticles. Pharmaceutics. 2018;10(4):267. doi:10.3390/pharmaceutics 10040267

64. Caldorera-Moore M, Guimard N, Shi L, Roy K. Designer nanoparticles: incorporating size, shape and triggered release into nanoscale drug carriers. Expert Opin Drug Deliv. 2010;7(4):479-495. doi:10.1517/17425240903579971

65. Bicudo RCS, Santana MHA. Production of hyaluronic acid (HA) nanoparticles by a continuous process inside microchannels: effects of non-solvents, organic phase flow rate, and HA concentration. Chem Eng Sci. 2012;84:134-141. doi:10.1016/j.ces.2012.08.010

66. Arduino I, Liu Z, Rahikkala A, et al. Preparation of cetyl palmitate-based PEGylated solid lipid nanoparticles by microfluidic technique. Acta Biomater. 2021;121(xxxx):566-578. doi:10.1016/j. actbio.2020.12.024

67. Damiati S, Kompella UB, Damiati SA, Kodzius R. Microfluidic devices for drug delivery systems and drug screening. Genes (Basel). 2018;9(2):103. doi:10.3390/genes 9020103

68. Laity P, Cassidy A, Skepper J, Jones B, Cameron R. Investigation into the intragranular structures of microcrystalline cellulose and pre-gelatinised starch. Eur J Pharm Biopharm. 2010;74(2):377-387. doi:10.1016/j.ejpb.2009.10.006

69. Coluccio ML, Perozziello G, Malara N, et al. Microfluidic platforms for cell cultures and investigations. Microelectron Eng. 2019;208:14-28. doi:10.1016/j.mee.2019.01.004

70. Grüll H, Langereis S. Hyperthermia-triggered drug delivery from temperature-sensitive liposomes using MRI-guided high intensity focused ultrasound. $J$ Control Release. 2012;161(2):317-327. doi:10.1016/j.jconrel.2012.04.041

71. Kim HJ, Huh D, Hamilton G, Ingber DE. Human gut-on-a-chip inhabited by microbial flora that experiences intestinal peristalsis-like motions and flow. Lab Chip. 2012;12 (12):2165-2174. doi:10.1039/c2lc40074j

72. Le TN, Nguyen VA, Bach GL, Tran LD, Cao HH. Design and fabrication of a PDMS-based manual micro-valve system for microfluidic applications. Adv Polym Technol. 2020;2020:1-7. doi:10.1155/ 2020/2460212

73. Khan IU, Serra CA, Anton N, Vandamme TF. Production of nanoparticle drug delivery systems with microfluidics tools. Expert Opin Drug Deliv. 2015;12(4):547-562. doi:10.1517/ 17425247.2015.974547

74. Liu D, Zhang H, Herranz-Blanco B, et al. Microfluidic assembly of monodisperse multistage $\mathrm{pH}$-responsive polymer/porous silicon composites for precisely controlled multi-drug delivery. Small. 2014;10 (10):2029-2038. doi:10.1002/smll.201303740

75. Zhang H, Liu D, Shahbazi MA, et al. Fabrication of a multifunctional nano-in-micro drug delivery platform by microfluidic templated encapsulation of porous silicon in polymer matrix. Adv Mater. 2014;26(26):4497-4503. doi:10.1002/adma.201400953

76. Li C, Boban M, Tuteja A. Open-channel, water-in-oil emulsification in paper-based microfluidic devices. Lab Chip. 2017;17 (8):1436-1441. doi:10.1039/C7LC00114B

77. Kim DY, Jin SH, Jeong SG, Lee B, Kang KK, Lee CS. Microfluidic preparation of monodisperse polymeric microspheres coated with silica nanoparticles. Sci Rep. 2018;8(1):1-11. doi:10.1038/s41598018-26829-z

78. Zhou W, Feng M, Valadez A, Li XJ. One-step surface modification to graft DNA codes on paper: the method, mechanism, and its application. Anal Chem. 2020;92(10):7045-7053. doi:10.1021/acs. analchem.0c00317 
79. Trouillon R, Gijs MAM. Dynamic electrochemical quantitation of dopamine release from a cells-on-paper system. RSC Adv. 2016;6 (37):31069-31073. doi:10.1039/c6ra02487d

80. Huang KS, Yang CH, Wang YC, Wang WT, Lu YY. Microfluidic synthesis of vinblastine-loaded multifunctional particles for magnetically responsive controlled drug release. Pharmaceutics. 2019;11 (5):212. doi:10.3390/pharmaceutics11050212

81. Amoyav B, Benny O. Microfluidic based fabrication and characterization of highly porous polymeric microspheres. Polymers (Basel). 2019;11:419. doi:10.3390/polym11030419

82. Vasiliauskas R, Liu D, Cito S, et al. Simple microfluidic approach to fabricate monodisperse hollow microparticles for multidrug delivery. ACS Appl Mater Interfaces. 2015;7(27):14822-14832. doi:10.1021/ acsami.5b04824

83. Zhang L, Chen Q, Ma Y, Sun J. Microfluidic methods for fabrication and engineering of nanoparticle drug delivery systems. ACS Appl Bio Mater. 2019;3(1):107-120. doi:10.1021/acsabm.9b00853

84. Xu Q, Hashimoto M, Dang TT, et al. Preparation of monodisperse biodegradable polymer microparticles using a microfluidic flow-focusing device for controlled drug delivery. Small. 2009;5 (13):1575-1581. doi:10.1002/smll.200801855

85. Maher S, Santos A, Kumeria T, et al. Multifunctional microspherical magnetic and $\mathrm{pH}$ responsive carriers for combination anticancer therapy engineered by droplet-based microfluidics. J Mater Chem B. 2017;5(22):4097-4109. doi:10.1039/C7TB00588A

86. Dashtimoghadam E, Mirzadeh H, Taromi FA, Nyström B. Microfluidic self-assembly of polymeric nanoparticles with tunable compactness for controlled drug delivery. Polymer (Guildf). 2013;54 (18):4972-4979. doi:10.1016/j.polymer.2013.07.022

87. Mu X, Gan S, Wang Y, Li H, Zhou G. Stimulus-responsive vesicular polymer nano-integrators for drug and gene delivery. Int J Nanomedicine. 2019;14:5415-5434. doi:10.2147/IJN.S203555

88. Chen W, Meng F, Cheng R, Zhong Z. pH-Sensitive degradable polymersomes for triggered release of anticancer drugs: a comparative study with micelles. J Control Release. 2010;142 (1):40-46. doi:10.1016/j.jconrel.2009.09.023

89. Paasonen L, Laaksonen T, Johans C, Yliperttula M, Kontturi K, Urtti A. Gold nanoparticles enable selective light-induced contents release from liposomes. J Control Release. 2007;122(1):86-93. doi:10.1016/j.jconrel.2007.06.009
90. Ganta S, Devalapally H, Shahiwala A, Amiji M. A review of stimuli-responsive nanocarriers for drug and gene delivery. J Control Release. 2008;126(3):187-204. doi:10.1016/j. jconrel.2007.12.017

91. Ranjan A, Jacobs GC, Woods DL, et al. Image-guided drug delivery with magnetic resonance guided high intensity focused ultrasound and temperature sensitive liposomes in a rabbit Vx2 tumor model. J Control Release. 2012;158(3):487-494. doi:10.1016/j. jconrel.2011.12.011

92. Meng L, Deng Z, Niu L, et al. A disposable microfluidic device for controlled drug release from thermal-sensitive liposomes by high intensity focused ultrasound. Theranostics. 2015;5(11):1203-1213. doi: $10.7150 /$ thno. 12295

93. Liu D, Zhang H, Cito S, et al. Core/shell nanocomposites produced by superfast sequential microfluidic nanoprecipitation; 2017. Available from: http://pubs.acs.org. Accessed January 6, 2017.

94. Morikawa Y, Tagami T, Hoshikawa A, Ozeki T. The use of an efficient microfluidic mixing system for generating stabilized polymeric nanoparticles for controlled drug release. Biol Pharm Bull. 2018;41(6):899-907. doi:10.1248/bpb.b17-01036

95. Wang J, Chen W, Sun J, et al. A microfluidic tubing method and its application for controlled synthesis of polymeric nanoparticles. $L a b$ Chip. 2014;14(10):1673-1677. doi:10.1039/c4lc00080c

96. Bazban-Shotorbani S, Dashtimoghadam E, Karkhaneh A, HasaniSadrabadi MM, Jacob KI. Microfluidic directed synthesis of alginate nanogels with tunable pore size for efficient protein delivery. Langmuir. 2016;32(19):4996-5003. doi:10.1021/acs. langmuir.5b04645

97. Chung JA, And DK, Erickson D. Electrokinetic microfluidic devices for rapid, low power drug delivery in autonomous microsystems. Lab Chip. 2008;8(2):330-338. doi:10.1021/acs.langmuir.5b04645

98. Santini JT, Richards AC, Scheidt R, Cima MJ, Langer R. Microchips as controlled drug-delivery devices. Angew Chemie Int Ed. 2000;39 (14):2396-2407. doi:10.1002/1521-3773(20000717)39
Drug Design, Development and Therapy

\section{Publish your work in this journal}

Drug Design, Development and Therapy is an international, peerreviewed open-access journal that spans the spectrum of drug design and development through to clinical applications. Clinical outcomes, patient safety, and programs for the development and effective, safe, and sustained use of medicines are a feature of the journal, which has also been accepted for indexing on PubMed Central. The manuscript management system is completely online and includes a very quick and fair peer-review system, which is all easy to use. Visit http://www. dovepress.com/testimonials.php to read real quotes from published authors. 